\title{
Ground Reaction Forces in Alternative Footwear during Slip Events
}

\author{
Harish Chander \\ Department of Kinesiology, Mississippi State University, 216 McCarthy Gym \\ Mississippi State, Mississippi 39762 \\ E-mail: hchander@colled.msstate.edu \\ John C. Garner (Corresponding author) \\ Department of Health, Exercise Science and Recreation Management, The University of Mississippi \\ Turner 219, University, Mississippi 38677 \\ E-mail: jcgarner@olemiss.edu \\ Chip Wade \\ Department of Industrial \& Systems Engineering, Auburn University \\ 3301-G Shelby Center, Auburn, Alabama 36849 \\ E-mail: 1rw0002@auburn.edu
}

Received: 3-03- 2015

Accepted: 18-04- 2015

Published: 30-04- 2015

doi:10.7575/aiac.ijkss.v.3n.2p.1

URL: http://dx.doi.org/10.7575/aiac.ijkss.v.3n.2p.1

\begin{abstract}
Slips, trips and falls are major causitive factors for occupational and non-occupational falls. Alternative footwear such has crocs and flip flops have been used in and around work places and communities that can be slip prone environments. The purpose of the study is to analyze the effects of alternative footwear [crocs (CC), flip-flops (FF)] and industry standard slip resistant shoes (LT) on ground reaction forces (GRFs) during slip events. Eighteen healthy male participants following a repeated measures design for each footwear condition, were tested for heel kinematics during normal dry surface gait (NG); unexpected slip (US), alert slip (AS) and expected slip (ES). A 3x4 repeated measures ANOVA was used to analyze the dependent vertical GRFs parameters (Mean Z-GRF and Peak Z-GRF) at $p=0.05$. Significant interactions between footwear and gait trials were found for Mean Z-GRF and significant main effect in gait trials for Peak Z-GRF were evident. On average significantly lower GRFs were seen in slip trials compared to normal gait. FF exhibited significantly lower GRFs during slip trials while LT demonstrated lower GRFs in normal gait. The reduced ground reaction forces during all slip events compared to normal gait can be attributed to the incomplete weight transfer on the slipping foot during the unexpected and alert slips and to the anticipation of the slippery environment in expected slips. Flip flops which had greater incidence of slips also demonstrated reduced GRFs compared to CC and LT during slip events, further suggesting incomplete weight transfer, while during normal gait, LT demonstrated reduced GRFs compared to alternative footwear owing to its cushioning midsole properties. The LT with lowest incidence of slips demonstrates to be the choice of footwear for maneuvering slippery flooring conditions and for reducing impact reaction forces during non-slippery flooring conditions.
\end{abstract}

Keywords: Slips, Falls, Alternative Footwear, Ground Reaction Forces, Perception of Slipperiness

\section{Introduction}

Increased probability of falls have been related to decrements in balance control and these falls are often a primary causative factor for injuries and disabilities in the general population as well as in the contemporary industrial population where postural stability is challenged with unfavorable and unfamiliar environment (Lin, Seol, Nussbaum \& Madigan, 2008). These falls are not always from an elevation and about $43 \%$ of the same level falls have been found to be triggered by slips (Courtney et al. 2001). Slips, trips and falls occur as a result of failure of normal locomotion and failure of attempts at equilibrium recovery following an induced imbalance (Davis, 1983; Gauchard, 2001). The coefficient of friction (COF) at the footwear-floor interface has been used as an applied approach in the prediction of outcome of slips. Under normal walking conditions the required or the utilized coefficient of friction (RCOF) have been shown to range from 0.17 to 0.20 (Redfern et al. 2001). When the required coefficient of friction exceeds the available coefficient of friction at the footwear-floor interface, the slip propensity increases (Redfern et al. 2001; Cham and Redfern, 2002b; Hanson et al, 1999; McGorry et al, 2010).

Ground reaction forces (GRF) during the stance phase of the gait cycle are influenced by the available coefficient of friction in the floor, the perception of slipperiness of the floor and the type of footwear used. A few kinematic and kinetic variables are very commonly used as outcome variables to interpret the slip propensity. The most commonly 
reported kinematic slip parameters include the heel slip distance, heel velocity and the foot-floor angle; joint angles of the ankle and the knee joints along with temporal-spatial parameters of gait including stride/step lengths, width of walking base, stride/step time and cadence; while kinetic parameters reported include center of pressure and ground reaction forces (Perkins, 1978; Strandberg \& Lanshammar, 1981; Redfern et al 2001). The ground reaction forces occurring immediately post heel strike is vital in the prediction of the slips and falls. The first peak in the shear force which occurs at about $19 \%$ of the gait cycle $(90-150 \mathrm{~ms}$ post heel strike) is the crucial time period during which most slips occur (Redfern et al. 2001). The highest shear forces occur during the heel contact and push-off phases of the gait cycle and considered as the points during which the highest incidence for a slip exists (Redferen et al. 2001; Redfern \& DiPasquale, 1997; Hanson et al, 1999). The general characteristics seen with ground reaction forces during slips are a reduction in the shear and normal forces, as the transfer of body weight to the supporting leg is not completed (Redfern et al. 2001). The changes in the gait variables in response to the perception of slippery hazardous conditions include a shorter step / stride length, thereby producing low heel velocities, smaller shear forces and lower required COF and lower GRFs during heel strike and push-off phases to reduce the likelihood of a slip. Anticipation of slippery walking trials in comparison to dry normal walking produced lower required coefficient of friction, reducing slip potential and failed to return to baseline normal dry walking values (Cham \& Redfern, 2002a). This reduction in the peak RCOFs were brought about by postural changes and adaptations during the gait cycle, with decreased step length, low impact GRFs and with significant changes in joint moments (Cham \& Redfern, 2002a; Cohen and Cohen, 1994; DiDomenico et al, 2007).

Both extrinsic factors such as the type of footwear ( $\mathrm{Li} \& \mathrm{Chen}, 2005 ; \mathrm{Li}, \mathrm{Wu} \& \mathrm{lin}, 2006$ ) and intrinsic factors such as the knowledge or anticipation of a slip (Cham \& Redfern, 2002a; Lockhart et al. 2007) have been shown to affect the outcome of a slip event. Extensive literature on kinematic gait analyses of barefoot and shod conditions during dry normal surface exists, which have focused on alternative open-toed footwear such as the flip-flops and slip-on footwear in comparison to close-toed shod conditions such as the athletic shoes or any shod condition that has a concealed foot with cushioning properties. Ground reaction forces in alternative footwear such as flip-flops, open-toed shoes and other shod conditions have been analyzed previously. Lower peak propulsive GRF and smaller loading rate of $1^{\text {st }}$ peak vertical GRF were reported in shoes compared to barefoot, sandals and flip-flops (Zhang et al. 2013) and reduced peak vertical ground reaction force was reported in shoes compared to barefoot (Yan et al. 2013). Similarly, in another study, flip flops were found to have the highest GRFs compared to barefoot walking (Shakoor et al. 2010). Although considerable amount of literature exists, analyzing the gait kinematics and kinetics in different footwear and shod conditions, the impact of these alternative footwear under slippery conditions haven't been fully addressed yet. The specific purpose of this paper is to analyze the impact of alternative footwear [Crocs with clogs (CC), Flip-Flops (FF) and Low Top Slip Resistant Shoe (LT)] under multiple gait conditions [Dry normal surface (NG); Unexpected Slip (US), Alert Slip (AS) and Expected Slip (ES)] on vertical ground reaction forces (Mean Z-GRF and Peak Z-GRF). Based on previous literature, we hypothesized that the vertical ground reaction forces (Z-GRF) will be greater for alternative footwear (CC \& FF) and minimized during slip trials.

\section{Methodology}

\subsection{Participants}

Eighteen healthy male participants [Age: $22.28 \pm 2.2$ years; Height: $177.66 \pm 6.9 \mathrm{~cm}$; Mass: $79.27 \pm 7.6 \mathrm{~kg}$ ] completed the study. Participants who had any history of musculoskeletal injuries, cardio-vascular abnormalities, and neurological disorders, vestibular disorders, under medications or any inability to walk and stand without support were excluded from the study. All participants were recruited through flyers approved by the University's Institutional Review Board (IRB). All participants read and signed the informed consent and also filled out the physical activity readiness questionnaire (PAR-Q) to rule out any of the above mentioned health complications and cleared for participation in the study.

\subsection{Instrumentation}

\subsubsection{D Motion Capture and Force Plates}

Vicon Nexus (Oxford, UK) 3D motion capture system with 12 infra-red T-series cameras was used to collect and analyze kinematic gait data. A lower body plug-in gait model from the Helen-Hayes marker system was used as for the participant configuration model. The motion capture system was calibrated every day prior to data collection and the kinematic data was sampled at $100 \mathrm{~Hz}$ and collected using the Vicon Nexus software. The variables of interest were collected with two force plates Bertec (Bertec Corporation, Columbus, OH) and AMTI (AMTI Force and Motion, Watertown, MA) embedded in the vinyl floored surface of the Applied Biomechanics Laboratory. The force plates are positioned and set up in such a way that, during gait trials across the walkway, the right leg will strike the AMTI force plate and the left leg will strike the Bertec force plate. The force plate data is collected through the Vicon Nexus system as an analog device and sampled at $1000 \mathrm{~Hz}$.

\subsubsection{Fall Arrest System}

A uni-track fall arrest system from Rigid Lines (Millington, TN); a lightweight horizontal rigid fall arrest track capable of supporting up to $900 \mathrm{lb}$ and installed with an inverted-U steel frame fixed to the laboratory floor was used as the safety fall arrest system to prevent any undesired falls. Participants were attached to the fall arrest track with the help of a back pack type harness system attaching to a moveable trolley inside the fall arrest track. The fall arrest track and the 
harness along with the trolley were connected by a pulley system that allowed the investigators to move the trolley on top of the walking participant so that, the participants were not leading the trolley nor was the trolley leading the participant. By doing so, the investigators were able to remove any pull or influence on the participants' gait while wearing the harness.

\subsubsection{Slippery Agent - Contaminant}

Industrial vegetable based glycerol mixed with water in the ratio of $75 \%$ glycerol and $25 \%$ water was used as the slippery agent. The $75 \%-25 \%$ ratio was chosen for the study based on previous literature and with initial practice sessions in the laboratory prior to starting data collection. During the slip gait trials or slip events, glycerol was applied and evenly distributed on the Bertec force plate, on which the left leg of all participants, irrespective of their dominant extremity, would make contact during the gait trials.

\subsection{Experimental Procedures}

All participants visited the Applied Biomechanics Laboratory four times, separated by a minimum of 24 hours. A description of the experimental procedures for each visit is provided below.

Day 1:The first visit was treated as a familiarization day, where all participants were exposed to the testing measures for gait trials. Informed consent was obtained from all participants following which they completed a physical activity readiness questionnaire (PAR-Q) and screened for any complications that might hinder them from completing the study. Anthropometric measurements such as height, weight, leg length, knee width, ankle width and foot/shoe size. Following this, participants were briefed on the walking gait conditions and were allowed to practice walking at their self-selected pace across the lab walkway. Next the participants were positioned and strapped inside the harness connecting to the trolley and ultimately to the fall arrest track. Participants also performed practice gait trials with the harness while the investigators moved the trolley in-sequence along with the participant. A number of practice gait trials were performed until the subjects walked normally and with the same speed. Finally, the participants were assured of the fall arrest system catching them in the case of an undue fall, initiated by the slip. All participants were also asked to refrain from any physical workload especially to their lower extremities in terms of resistance or aerobic training and were also asked to avoid any pain medications until all their testing days were completed.

Day 2, Day 3 and Day 4: Visits 2, 3 and 4 were again separated by a minimum of 24 hours and treated as experimental testing days. These experimental testing days followed the same testing protocol except with different alternative footwear (CC, FF \& LT) which were chosen and provided to the participant using a counter balance design to remove order effects.

Picture 1: Crocs $(\mathrm{CC})$

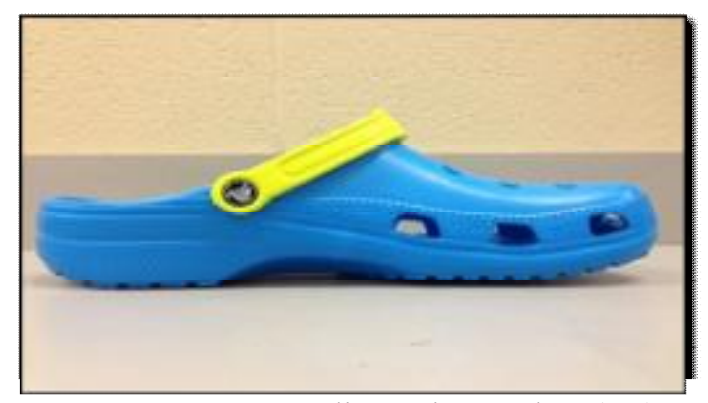

Picture 3: Low Top Slip Resistant Shoe (LT):
Picture 2: Flip Flops (FF)

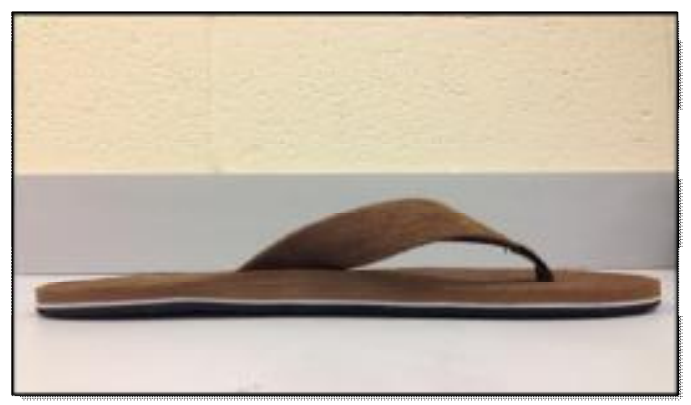

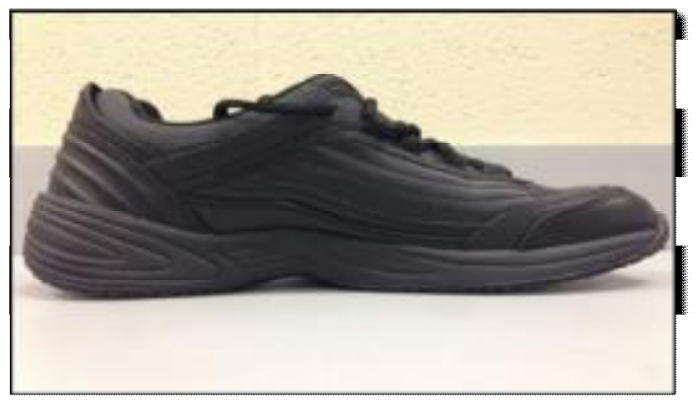

Experimental Testing: The experimental testing session began with the participant wearing the footwear given to them and reflective markers were placed on the participant's lower extremity and on the footwear following a lower body plug-in gait model from the Helen-Hayes system. Participants were then strapped in the harness system and had a series of practice gait trials across the vinyl floored testing surface under dry non slip conditions to get accustomed with the gait trials and the testing environment at a self-selected speed of walking. These practice gait trials were also used to make sure that the participants strike the center of the force plate with both their feet, unintentionally at their normal walking pattern and pace and to avoid any intentional modification of their step lengths during the data collection 
procedure. Following the initial practice gait trials, data collection was done for five normal dry gait trials with no breaks or stops between the gait trials with the instruction "walk as normally as possible with the same speed". With the completion of the fifth normal dry gait trial, participants still walked with the same pattern and speed, but at the end of all further gait trials, the participants took 30-45 second breaks facing away from the walking surface and listened to music played on noise-cancellation headphones, which would take away the knowledge of the potential slip trial.

Following a repeated number of gait trials under normal dry conditions, one particular trial was chosen randomly to be the unexpected slip (US) trial and the contaminant was applied to the force plate without the participant's knowledge. Participants were still given the same walking instruction to ensure that the walking trial will be treated as an unexpected slip event. On completion of the US, participants were allowed to rest briefly and the footwear removed for cleaning the contaminant. The force plate was also cleaned with a dry-wet vacuum and soap water and dried completely and made ready for the next gait trials. Participants then performed multiple normal dry gait trials with the same 30-45 second breaks and once a normal gait pattern resumed, participants were given the instruction that all of the following trials "may or may not be slippery". Multiple gait trials with the same protocol and instructions were performed in succession and one trial was randomly chosen to be the alert slip (AS) trial, where the contaminant was applied again without the knowledge of the participant, but differed from the US in terms of the instruction given to the participant. Finally, with the completion of NG, US and AS, participants visually saw the application of the contaminant on the force plate for one last walking trial and were given the instruction that the following trial "will be slippery" and treated as an expected slip (ES) trial.

\subsection{Data Analysis}

The analog kinetic force plate data measures were analyzed using the Vicon Nexus software. The raw data was filtered using a Butterworth fourth order filter with zero lag with a cut of frequency of $300 \mathrm{~Hz}$ and exported as excel files for further analyses. Vicon Nexus software was used to determine the moment of heel strike and toe off phase of the left leg during the gait trials to determine the stance phase beginning and ending of the stance phase. The Ground reaction force in the vertical (Z) direction (Z-GRF) from the Bertec force plate during the left leg's stance phase was used for the kinetic measure. Mean Z-GRF and Peak Z-GRF were calculated from the exported excel files.

\subsection{Statistical Analysis}

A within-subjects repeated measures of analysis of variance (repeated measures ANOVA) was performed to compare footwear conditions across gait trials. A 3x4 [3 Footwear (CC, FF, LT) x 4 Gait Trials (NG, US, AS, ES)] repeated measures ANOVA was used to analyze the dependent kinetic variables of Mean Z-GRF and Peak Z-GRF individually for footwear $\mathrm{x}$ gait trial interaction and main effect significance. The dependent variables were tested initially for the footwear $\mathrm{x}$ gait trial interaction, and if a significant interaction existed, the main effects of footwear and gait trials were ignored and pairwise comparisons of the simple main effects for the existing significant interaction was performed using the Sidak Bonferroni multiple comparisons procedure, independently for footwear effect and gait trials effect. For all analyses, alpha level was set a priori at $\mathrm{p}=0.05$ and all statistical analyses was performed using the SPSS 21 statistical software package.

\section{Results}

The repeated measures ANOVA revealed significant interactions between footwear and gait trials for Mean Z-GRF. Significant interaction between footwear and gait trials existed for Mean Z-GRF at F $(3.163,53.766)=4.236, p=$ $0.008, \eta_{p}{ }^{2}=0.199$ (Fig.1). Pairwise comparisons using the Sidak Bonferroni correction was performed to analyze the simple main effects across both factors of footwear and gait trials for Mean Z-GRF. Pairwise comparisons for simple main effects for footwear revealed significant differences for CC between NG and US at $p=0.019$; between NG and AS $p=0.015$; and between NG and ES at $p=0.0005$, with significantly greater Mean Z-GRF for NG compared to US, AS and ES; and for FF between NG and US at $p=0.001$; between NG and AS $p=0.011$; and between NG and ES at $p$ $=0.0005$, with significantly greater Mean Z-GRF for NG compared to US, AS and ES; for LT between NG and ES at $p$ $=0.018$, with significantly greater Mean Z-GRF for NG compared to ES. Pairwise comparisons for simple main effects for gait trials revealed significant differences for NG between CC and LT at $p=0.008$, with significantly greater Mean Z-GRF for CC compared to LT; for US between CC and FF at $p=0.02$ with significantly greater Mean Z-GRF for CC compared to FF; for AS between FF and LT at $p=0.014$, with significantly greater Mean Z-GRF for LT compared to FF. No significant differences existed for ES across all footwear. No significant footwear $\mathrm{x}$ gait trials interaction existed for Peak Z-GRF and for main effect in footwear. However, there was a significant difference in main effect for gait trials at $\mathrm{F}(3,51)=7.240, p=0.0005, \eta_{p}{ }^{2}=0.299$ (Fig.2). Pairwise comparisons using a Bonferroni correction revealed significant differences between NG and AS $\mathrm{p}=0.001$ and between NG and ES at $p=0.0005$, with significantly greater Peak Z-GRF for NG compared to AS and ES. 


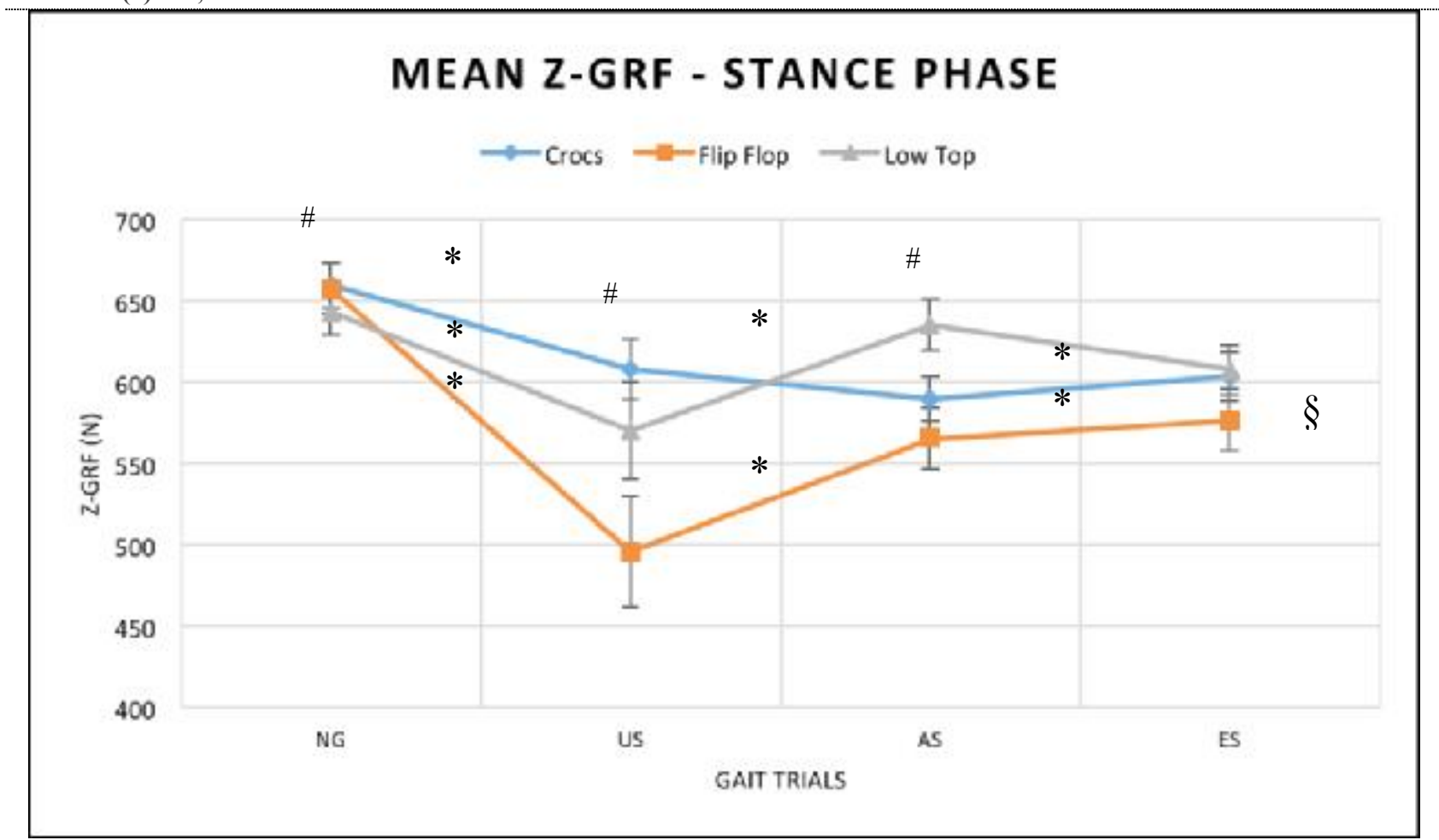

Figure 1. Mean Z-Ground Reaction Force (N) during stance phase for Crocs, Flip-Flops and Low Top Slip Resistant Shoe during normal gait (NG), unexpected slip (US), alert slip (AS) and expected slip (ES) events

$\S$ denotes significant interaction; * denotes significant difference for footwear across gait trials and \# denotes significant difference for gait trials across footwear. Bars represent standard error. All differences were significant at alpha level $p=0.05$.

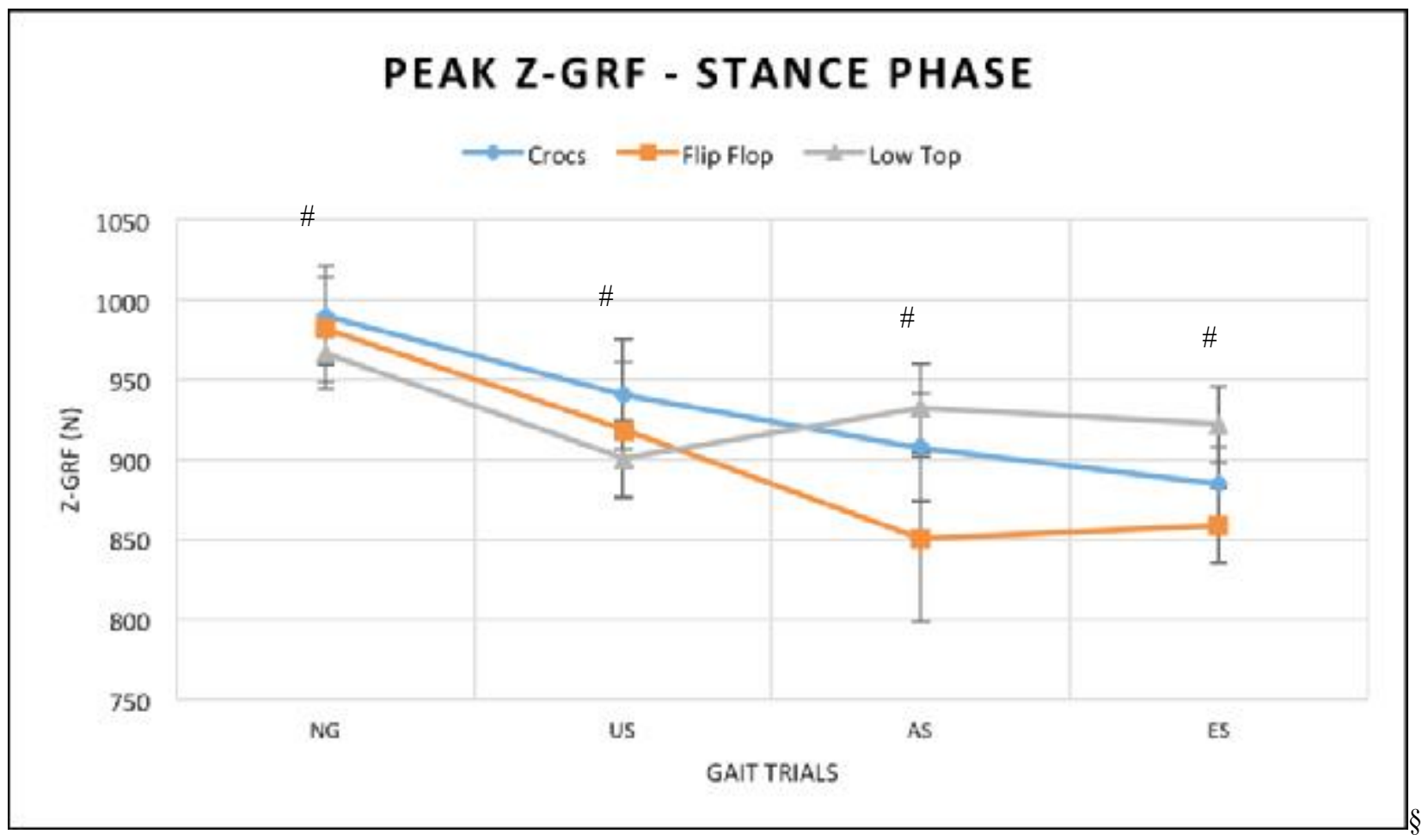

Figure 2. Peak Z-Ground Reaction Force (N) during stance phase for Crocs, Flip-Flops and Low Top Slip Resistant Shoe during normal gait (NG), unexpected slip (US), alert slip (AS) and expected slip (ES) events

denotes significant interaction; * denotes significant difference for footwear across gait trials and \# denotes significant difference for gait trials across footwear. Bars represent standard error. All differences were significant at alpha level $p=0.05$. 


\section{Discussion}

The purpose of this study was to analyze the effect of alternative footwear, [Crocs with Clogs (CC), Flip-Flops (FF) and Low Top Slip Resistant Shoe (LT)] on vertical ground reaction forces (Mean Z-GRF and Peak Z-GRF) during non-slip and slip gait trials [Normal Dry Gait (NG), Unexpected Slip (US), Alert Slip (AS) and Expected Slip (ES)]. The findings from the study demonstrate significant differences in both mean and peak vertical ground reaction forces during the stance phase. On average, lower ground reaction forces were seen in all slip trials compared to normal dry surface gait. However, footwear differences were varied and were dependent upon the gait trial being either slippery or non-slippery. The specific purpose of this paper is to analyze the impact of alternative footwear on vertical ground reaction forces during slip events. The incidence of the slips and slip induced falls across different slip trials and across types of footwear are reported elsewhere, with significantly greater incidence of slips in alternative footwear (CC \& FF) compared to LT and with significantly greater magnitude of slips in US followed by AS and ES compared to NG. Significant interactions between footwear and gait trials existed for Mean Z-GRF and main effect significance for gait trials existed for Peak Z-GRF, suggesting the influence of both footwear and gait trial influence in the outcome of vertical ground reaction forces. Kinetic force plate data from the current study demonstrate significantly lower mean and peak ground reaction forces for all slip trials (US, AS, ES) compared to the dry normal gait (NG) for all footwear, which can be attributed to the incomplete transfer of weight to the stance/support extremity during unexpected slips and due to the anticipation of the slippery environment in alert and expected slips. Individual footwear variations in each of the gait trial were varied where greater mean Z-GRF was seen in CC compared to LT in NG and compared to FF in US. AS exhibited greater mean GRF in LT compared to FF, while no significant differences existed for ES, where slips were minimal irrespective of the footwear used and subsequently ground reaction forces were similar across all footwear during ES.

\subsection{Extrinsic Factors - Impact of footwear design characteristics}

The footwear's geometrical design characteristics have been shown to affect human balance and gait (Chander et al. 2014; Perry, Radtke \& Goodwin 2007; Menant et al. 2008; Divert et al. 2005; Bohm \& Hosl 2010) and especially with the growing usage of alternative footwear such as the thong-styled flip flops and open-toed sandals, several studies have focused on their impact on the biomechanics of human gait (Zhang et al. 2013; Shroyer et al. 2010). The impact of flip flops on ground reaction forces reported from previous studies have shown an increased peak vertical ground reaction force in flip flops (Shakoor et al. 2010; Zhang et al. 2013) compared to other shod conditions and have attributed their findings to the reduced shock attenuation capabilities of the flip flops. The flip flop has also been reported to have a modified "touch down geometry" that can influence ground reaction forces in stance phase (De Wit et al. 2000; Shroyer et al. 2010). The current study exhibited greater Mean Z-GRF in CC compared to LT, which again may be attributed to the lower shock attenuation capabilities of the crocs and the absence of a cushion type sole. Although the CC and FF had similar Mean Z-GRFs, significant differences were found only between CC and LT, but not between FF and LT. These findings were solely under dry normal gait conditions. The impact of these alternative footwear under slippery conditions is still unknown. The current study demonstrated significantly lower Mean Z-GRF for FF compared to CC in US and LT in AS, which may be attributed to the greater incidence of slips in FF with incomplete transfer of body weight to the lower extremity undergoing slips (Redfern et al. 2001), while individuals during the ES were able to minimize the slips and did not have significant differences between footwear. Across gait trials, both Mean and Peak ZGRF were significantly lower in all slip trials compared to normal dry gait trial in all footwear conditions. The lower GRFs in slippery conditions can be related to the greater incidence of slips and due to the body weight not being completely transferred to the slipping extremity (Redfern et al. 2001) rather than to the design or geometric features of the footwear.

\subsection{Intrinsic Factors - Impact of perception and anticipation of slips}

The perception and anticipation of a slippery environment has been shown as a vital intrinsic factor in predicting the outcome of a slip event. The prior knowledge and anticipation of a slippery floor allows the individuals to reduce the potential slips by making adaptations to the biomechanics of gait (Cham \& Redfern, 2002a; Lockhart et al. 2007). These gait adaptations which attempt to minimize the incidence of slips and slip induced falls include a shorter step length, a reduced foot floor angle and creating a more flat foot placement at heel strike and subsequently reducing the heel contact velocity, and also minimize the normal and shear forces during stance phase and thereby also lowering the required coefficient of friction when maneuvering slippery flooring conditions (Lockhart et al. 2007; Redfern et al. 2001; Cham and Redfern, 2002a; Moyer et al. 2006). Based on the results from our study, the reduction in the ground reaction forces during the expected slip (ES) across all footwear can be attributed to these gait modifications that occur under slippery conditions. This was also evident by least number of slip incidences in the ES. However, the reduced Mean and Peak Z-GRF during the US cannot be treated as anticipatory gait modifications. During these unexpected slippery gait trials the individuals had either no knowledge of the slippery flooring conditions and the highest incidence of slips. Hence, the reductions in the GRFs during US could only be attributed to the incomplete transfer of the body weight on to the supporting and slipping lower extremity (Redfern et al. 2001). The AS gait condition, provided the individuals with a warning of a slippery floor, but they were still unaware of which trial would be slippery. Alert slips exhibited the second highest incidence of slips followed by unexpected slips. Hence, the reduction in the GRFs may be attributed to either one or both of the above mentioned reasons. 


\section{Conclusion}

Based on the results from the current study, the interaction between the type of footwear and the non-slip and slip gait trial conditions, suggest the influence of both footwear design features and perception or anticipation of slippery flooring conditions on the vertical ground reaction forces. The GRFs during slip trials were reduced compared to normal dry gait conditions across all footwear due to incomplete weight transfer on the slipping foot during unexpected and alert slips trials and in an attempt to minimize the required coefficient of friction and prevent slips, with anticipation of slips during expected slips. Flip flops which had greater incidence of slips also demonstrated reduced GRFs compared to CC and LT during slip events, further suggesting incomplete weight transfer, while during normal gait, LT demonstrated reduced GRFs compared to alternative footwear owing to its cushioning midsole properties. The LT had better performance in preventing slips and given the conditions, the low top slip resistant shoe proves to be the choice of footwear for maneuvering slippery flooring conditions. Even though the alternative footwear serves for comfort and easy donning, it might not be the choice of footwear to prevent slips and slip induced falls. Future research should focus on the interactions of these alternative footwear and slippery conditions with an additional physiological workload or under muscular fatigue and exertion.

\section{References}

Bohm, H, \&Hosl, M. (2010). Effect of boot shaft stiffness on stability joint energy and muscular co-contraction during walking on uneven surface. Journal of Biomechanics, 43, 2467-2472.

Chander, H., Garner, J. C., \& Wade, C. (2014). Impact on balance while walking in occupational footwear. Footwear Science, 6(1), 59-66.

Cham, R., Redfern, M.S., (2002a). Changes in gait when anticipating slippery floors. Gait \& Posture 15, 159-171.

Cham, R., Redfern, M.S., (2002b). Heel contact dynamics during slip events on level and inclined surfaces. Safety

Science. 40, 559-576.

Cohen, H.H., Cohen, D.M., (1994). Perceptions of walking surface slipperiness under realistic conditions, utilizing a slipperiness rating scale. Journal of Safety Research. 25(1), 27-31.

Courtney, T. K., Sorock, G. S., Manning, D. P., Collins, J. W. and Holbein-Jenny, M. A. (2001). Occupational slip, trip, and fall-related injuries can the contribution of slipperiness be isolated? Ergonomics, 44, 1118- 1137.

Davis, P. R. (1983). Human factors contributing to slips, trips and falls. Ergonomics, 26(1), 51-59.

De Wit, B., De Clercq, D., \& Aerts, P. (2000). Biomechanical analysis of the stance phase during barefoot and shod running. Journal of Biomechanics, 33(3), 269-278.

DiDomenico, A., McGorry, R. W., \& Chang, C. C. (2007). Association of subjective ratings of slipperiness to heel displacement following contact with the floor. Applied Ergonomics, 38(5), 533-539

Divert, C., Mornieux, G., Baur, H., Mayer, H., Belli, A. (2005). Mechanical Comparison of Barefoot and Shod

Running. International Journal of Sports Medicine, 26, 593 - 598.

Gauchard, G., Chau, N., Mur, J. M., \& Perrin, P. (2001). Falls and working individuals: role of extrinsic and intrinsic factors. Ergonomics, 44(14), 1330-1339.

Hanson, J. P., Redfern, M. S., \&Mazumdar, M. (1999). Predicting slips and falls considering required and available friction. Ergonomics, 42(12), 1619-1633.

Li, K. W., \& Chen, C. J. (2005). Effects of tread groove orientation and width of the footwear pads on measured friction coefficients. Safety Science, 43(7), 391-405.

Li, K. W., Wu, H. H., \& Lin, Y. C. (2006). The effect of shoe sole tread groove depth on the friction coefficient with different tread groove widths, floors and contaminants. Applied Ergonomics, 37(6), 743-748.

Lin D, Seol H, Nussbaum M, Madigan M (2008). Reliability of COP- based postural sway measures and age-related differences. Gait \& Posture, 28, 337-342

Lockhart, T. E., Spaulding, J. M., \& Park, S. H. (2007). Age-related slip avoidance strategy while walking over a known slippery floor surface. Gait \& Posture, 26(1), 142-149.

McGorry, R. W., DiDomenico, A., \& Chang, C. C. (2010). The anatomy of a slip: Kinetic and kinematic characteristics of slip and non-slip matched trials. Applied Ergonomics, 41(1), 41-46.

Menant, J, Perry, S, Steele, J, Menz, H,Munro, B, Lord, S (2008). Effects of Shoe Characteristics on Dynamic Stability When Walking on Even and Uneven Surfaces in Young and Older People. Arch Phys Med Rehabil; 89, 1970-6.

Moyer, B. E., Chambers, A. J., Redfern, M. S., \& Cham, R. (2006). Gait parameters as predictors of slip severity in younger and older adults. Ergonomics, 49(4), 329-343.

Perkins, P. J. 1978, Measurement of slip between the shoe and ground during walking, in Walkway Surfaces:

Measurement of Slip Resistance, ASTM STP 649, Philadelphia, PA.

Perry S, Radtke A, Goodwin C (2007). Influence of footwear midsole material hardness on dynamic balance control during unexpected gait termination. Gait \& Posture 25, 94-98.

Redfern, M. S., Cham, R., Gielo-Perczak, K., Grönqvist, R., Hirvonen, M., Lanshammar, H.,\& Powers, C. (2001).

Biomechanics of slips. Ergonomics, 44(13), 1138-1166.

Redfern, M.S., \&DiPasquale, J. (1997). Biomechanics of descending ramps. Gait \& Posture, 6(2), 119-125.

Shakoor, N., Sengupta, M., Foucher, K. C., Wimmer, M. A., Fogg, L. F., \& Block, J. A. (2010). Effects of common footwear on joint loading in osteoarthritis of the knee. Arthritis Care \& Research, 62(7), 917-923. 
Shroyer, J. F., \& Weimar, W. H. (2010). Comparative analysis of human gait while wearing thong-style flip-flops versus sneakers. Journal of the American Podiatric Medical Association, 100(4), 251-257.

Strandberg, L. and Lanshammar, H. (1981). The dynamics of slipping accidents, Journal of Occupational Accidents, 3, 153-162.

Yan, A. F., Sinclair, P. J., Hiller, C., Wegener, C., \& Smith, R. M. (2013). Impact attenuation during weight bearing activities in barefoot vs. shod conditions: a systematic review. Gait \& Posture, 38(2), 175-186.

Zhang X, Paquette MR \& Zhang S: A comparison of gait biomechanics of flip-flops, sandals, barefoot and shoes. (2013). Journal of Foot and Ankle Research, 45(6). 